\title{
Role of inducible nitric oxide synthase in trinitrobenzene sulphonic acid induced colitis in mice
}

\author{
D-M McCafferty, M Miampamba, E Sihota, K A Sharkey, P Kubes
}

\begin{abstract}
Background-Studies using inhibitors of nitric oxide synthase (NOS) to date are inconclusive regarding the role of inducible NOS (iNOS) in intestinal inflammation.

Aims-(1) To examine the role of iNOS in the development of chronic intestinal inflammation; (2) to identify the cellular source(s) of iNOS.

Methods-Colitis was induced by an intrarectal instillation of trinitrobenzene sulphonic acid (TNBS, $60 \mathrm{mg} / \mathrm{ml}, 30 \%$ ethanol), in wild type (control) or iNOS deficient mice. Mice were studied over 14 days; the colons were scored for injury and granulocyte infiltration was quantified. Blood to lumen leakage of ${ }^{51} \mathrm{Cr}$-EDTA was measured as a quantitative index of mucosal damage.
\end{abstract}

Results-At 24 and 72 hours, iNOS deficient mice had significantly increased macroscopic inflammation compared with wild type mice. Granulocyte infiltration increased significantly at 24 hours and remained elevated in iNOS deficient mice at 72 hours, but significantly decreased in controls. However, by seven days post-TNBS macroscopic damage, microscopic histology, granulocyte infiltration, and mucosal permeability did not differ between wild type and iNOS deficient mice. A four- to fivefold increase in iNOS mRNA was observed in wild type mice at 72 hours and seven days postTNBS and was absent in iNOS deficient mice. Immunohistochemistry techniques showed that iNOS expression was predominantly localised in neutrophils, with some staining also in macrophages.

Conclusions-These results suggest that leucocyte derived iNOS ameliorates the early phase, but does not impact on the chronic phase of TNBS induced colitis despite the presence of iNOS.

(Gut 1999;45:864-873)

Keywords: nitric oxide synthase; neutrophils; epithelial permeability; inflammatory bowel disease

Nitric oxide (NO) has received considerable attention regarding its role in various inflammatory conditions including the idiopathic inflammatory bowel diseases, ulcerative colitis, and Crohn's disease. ${ }^{1-3}$ Basal levels of nitric oxide are produced constitutively from nitric oxide synthase (NOS) associated with vascular endothelium (ecNOS or NOS-3) and present in nerves (bNOS or NOS-1). ${ }^{4}$ However, it is the inducible isoform of NOS (iNOS or NOS-2), capable of high output NO production in a calcium independent manner, which has been implicated in the pathophysiology of intestinal diseases. ${ }^{56}$

A pathophysiological role for $\mathrm{NO}$ derived from iNOS, has been suggested from the use of NOS inhibitors in experimental models of colitis. Inhibition of NO production was originally reported to ameliorate intestinal injury. ${ }^{6}{ }^{7}$ Since then, an array of pharmacological studies have supported a beneficial effect, ${ }^{5}$ shown little ${ }^{89}$ or no ${ }^{10}$ effect, or even shown an exacerbation of experimentally induced inflammation. ${ }^{11}$ However, because of the relative lack of specificity of the inhibitors used in these studies, these results must be viewed with caution. For example, although aminoguanidine is a more selective inhibitor of iNOS than cNOS (10-100 times $\left.{ }^{12}\right)$, it has effects on vascular function in vivo through an action on cNOS, ${ }^{13}$ and can also act as an inhibitor of histaminase. ${ }^{14}$ Therefore, studies using inhibitors of NOS to date are inconclusive regarding the role of iNOS in intestinal inflammation, partially because of relatively different levels of iNOS inhibition as well as non-specificity.

Recent advances in recombinant DNA technology have enabled the generation of mice which specifically lack the iNOS gene. ${ }^{15}$ These mice have normal levels of constitutive $\mathrm{NO}$ but are unable to produce any NO from the inducible isoform. In this study, we induced a chronic experimental colitis, in both wild type and iNOS deficient mice, by using the hapten 2,4,6-trinitrobenzene sulphonic acid (TNBS). ${ }^{16}{ }^{17}$ This is a well established model of experimental colitis, which has an immunological component, and is known to develop into a chronic intestinal inflammation approximately seven days after the induction of colitis. ${ }^{16}{ }^{17}$ The first aim of this study was therefore to determine the role of iNOS in experimentally induced chronic intestinal

Abbreviations used in this paper: bNOS, brain nitric oxide synthase; $\mathrm{cpm}_{\mathrm{pr}}$, counts per minute per $\mathrm{ml}$ perfusate; $\mathrm{cpm}_{\mathrm{p}}$, counts per minute per ml plasma; ecNOS, endothelial constitutive nitric oxide synthase; FITC, fluorescein isothiocyanate; GAPDH,

glyceraldehyde-3-phosphate dehydrogenase; iNOS, inducible nitric oxide synthase; MPO,

myeloperoxidase; NO, nitric oxide; NOS, nitric oxide synthase; PBS, phosphate buffered saline; PBS-T, PBS-Triton X-100; RT-PCR, reverse transcriptase polymerase chain reaction; TNBS, trinitrobenzene sulphonic acid. 
inflammation. Secondly, neutrophils, macrophages, epithelial cells, endothelial cells, and neurones have all been implicated as potential cellular sources of iNOS. Thus, we also endeavoured to identify the cellular source of iNOS in TNBS induced colitis using double labelling immunohistochemical techniques.

\section{Materials and methods}

Mice deficient in iNOS were generated by gene targeting in embryonic stem cells as described previously ${ }^{15}$ and obtained from Dr J Mudgett (Merck Research Laboratories, Rathway, New Jersey, USA). The mutant mice used for these experiments were from a mixed background of C57B16 × 129Sv/Ev. Appropriate wild type controls were used (Jackson Laboratories, Bar Harbor, Maine, USA). All animals were generated in specific pathogen free facilities and removed to conventional facilities for at least two weeks prior to experimentation. All experimental procedures were approved by the Animal Care Committee of the University of Calgary and conformed to the guidelines established by the Canadian Council for Animal Care.

EXPERIMENTAL COLITIS

Male or female mice weighing 20-30 g were used in all experiments. Colitis was induced by intrarectal administration of $0.1 \mathrm{ml}$ trinitrobenzene sulphonic acid (TNBS; $60 \mathrm{mg} / \mathrm{ml}$ in $30 \%$ ethanol), through a trochar needle approximately $3-4 \mathrm{~cm}$ proximal to the anus. This model has been described previously in detail. ${ }^{16}{ }^{17}$ Healthy (no treatment), wild type or iNOS deficient mice were also studied as controls. Animals were killed one, three, seven, or 14 days after the induction of colitis.

ANALYSIS OF INOS MRNA EXPRESSION BY RT-PCR Colon tissue samples from mice one, three, seven, and 14 days post-TNBS were rinsed in saline, weighed, and placed in guanidinium isothiocyanate to extract total RNA. ${ }^{18}$ The final RNA concentrations were determined by absorbance using a GeneQuant spectrophotometer (Pharmacia, Piscataway, New Jersey, USA). The reverse transcriptase (RT) and polymerase chain reaction (PCR) steps were performed as described by Wong et al. ${ }^{19}$ Briefly, complementary DNA (cDNA) was generated using a RT reaction by incubating $2 \mu \mathrm{g}$ of total RNA, 1× PCR buffer (10 mM Tris-HCl, pH 9.0, $50 \mathrm{mM} \mathrm{KCl,} 1.5 \mathrm{mM} \mathrm{MgCl}), 1 \mathrm{nM}$ each of deoxynucleotide triphosphates (dATP, dGTP, dCTP, and dTTP), 30 units of placental ribonuclease inhibitor (RNA guard, Pharmacia, Piscataway, New Jersey, USA), 200 units of Superscript reverse transcriptase (GIBCO-BRL, Burlington, Ontario, Canada), and $2000 \mathrm{pmol}$ of random hexamer oligodeoxynucleotides (Pharmacia, Piscataway, New Jersey, USA). Reaction mixtures were preincubated for 10 minutes at $21^{\circ} \mathrm{C}$ prior to DNA synthesis. The RT reactions were carried out for 50 minutes at $42^{\circ} \mathrm{C}$ and were heated to $95^{\circ} \mathrm{C}$ for five minutes to terminate the reaction. Reactions were performed in an Amplitron I
Thermal Cycler (Barnstead/Thermolyne, Dubuque, Iowa, USA).

Multiple PCR steps were performed using the primer dropping method as described by Wong et $a l^{19}$ in $50 \mu \mathrm{l}$ reaction volumes containing $2 \mu \mathrm{l}$ of RT reaction product as template DNA, $1 \times$ PCR buffer, $80 \mu \mathrm{M}$ of each dinucleotide, and 20 pmol of each specific $5^{\prime}$ and 3 ' starter primers. The following primer sequences (5'-3') were used for iNOS: the sense TCACTGGGACAGCACAGAAT and antisense TGTGTCTGCAGATGTGCTGA with a final PCR product size of 499 base pairs (bp). Glyceraldehyde-3-phosphate dehydrogenase (GAPDH) cDNA was coamplified as an internal control using the following primer sequences (5'-3'): sense CGGAGTCAACGGATTTGGTCGTAT and antisense AGCCTTCTCCATGGTGGTGAAGAC ${ }^{14}$ with a final PCR product size of $302 \mathrm{bp}$. Two units of Taq DNA polymerase (Pharmacia) were added to each tube during the first denaturation step and equal aliquots of secondary primer sets (20 pmol GAPDH) were added to the appropriate cycle number by the dropping method. Each PCR cycle consisted of a heat denaturation step at $94^{\circ} \mathrm{C}$ for one minute, a primer annealing step at $55^{\circ} \mathrm{C}$ for 30 seconds, and a polymerisation step at $72^{\circ} \mathrm{C}$ for one minute in an Amplitron I Thermal Cycler (Barnstead/Thermolyne). PCR cycle numbers were chosen to ensure that the amplification of PCR products was in the exponential range: 26 cycles for iNOS and 22 for GAPDH. Aliquots of PCR products (approximately $10 \mu \mathrm{l}$ ) equalised to given equivalent signals from the GAPDH mRNA were electrophoresed through $2 \%$ agarose gels (Ultrapure, Pharmacia) containing $0.5 \mu \mathrm{g} / \mathrm{ml}$ ethidium bromide. Gels were visualised under ultraviolet light and photographed with Kodak film. The optical density of each band for iNOS and GAPDH was quantified using NIH image 1.6 software and the results expressed as a ratio of iNOS to GAPDH.

ASSESSMENT OF SEVERITY OF COLITIS

Mice were killed by cervical dislocation, the colon excised, and the severity of colonic damage assessed using criteria previously established for TNBS induced colitis (table 1). ${ }^{20}$ This scoring system includes features of clinical colitis, macroscopically visible damage, the presence and severity of adhesions, the presence of diarrhoea (defined as loose, watery stool), presence of stricture formation, and maximal bowel wall thickness in millimetres. After gross macroscopic scoring, samples of colonic tissue were fixed in neutral buffered formalin and processed for subsequent histological examination. In addition samples of colon were taken for estimation of myeloperoxidase activity and immunohistochemical studies as described below.

HISTOLOGICAL SCORING

After overnight fixation in formalin, tissues were dehydrated (graded alcohols) and cleared (xylene) before being embedded in paraffin wax. Sections of tissue were cut and stained 
Table 1 Criteria used to assess macroscopic damage in trinitrobenzene sulphonic acid (TNBS) induced inflammation in mouse colon

\begin{tabular}{|c|c|}
\hline Score & Parameter \\
\hline 0 & No damage \\
\hline 1 & Hyperaemia without ulcers \\
\hline 2 & $\begin{array}{l}\text { Hyperaemia and thickening of bowel wall without } \\
\text { ulcers }\end{array}$ \\
\hline 3 & One site of ulceration without bowel wall thickness \\
\hline 4 & Two or more sites of ulceration or inflammation \\
\hline 5 & $0.5 \mathrm{~cm}$ of inflammation and major damage \\
\hline $6-10$ & $\begin{array}{l}1 \mathrm{~cm} \text { of major damage. The score is increased by } 1 \\
\text { for every } 0.5 \mathrm{~cm} \text { of damage observed to a maximum } \\
\text { of } 10\end{array}$ \\
\hline 0 or 1 & Absence or presence of diarrhoea \\
\hline 0 or 1 & Absence or presence of stricture \\
\hline 0,1 , or 2 & Absence or presence (mild or severe) of adhesions \\
\hline $\mathrm{mm}$ & Maximum bowel wall thickness in $\mathrm{mm}$ \\
\hline
\end{tabular}

On examination of the colon, a score for the extent of ulcerative damage was awarded. Added to this value was the score for presence or absence of diarrhoea, stricture, and adhesions, plus maximal bowel wall thickness in $\mathrm{mm}$. Adapted from Morris $e t$ $a .^{20}$

with haematoxylin and eosin and the sections scored blindly. Histological scoring was based on a semiquantitative scoring system where features were graded as follows: extent of destruction of normal mucosal architecture $(0$, normal; 1, 2, and 3, mild, moderate, and extensive damage respectively), presence and degree of cellular infiltration ( 0 , normal; 1,2 , and 3 , mild, moderate, and transmural infiltration respectively), extent of muscle thickening ( 0 , normal; 1,2 , and 3 , mild, moderate, and extensive thickening respectively), presence or absence of crypt abscesses (0, absent; 1, present) and the presence or absence of goblet cell depletion ( 0 , absent; 1 , present). The scores for each feature were summed with a maximum score possible of 11 .

\section{DETERMINATION OF TISSUE MYELOPEROXIDASE}

ACTIVITY

Samples of distal colon were weighed, frozen on dry ice, and processed for determination of myeloperoxidase (MPO) activity. Myeloperoxidase is an enzyme found in cells of myeloid origin, and has been used extensively as a biochemical marker of granulocyte (mainly neutrophil) infiltration into gastrointestinal tissues. ${ }^{20}{ }^{21}$ The samples were stored at $-20^{\circ} \mathrm{C}$ for no more than one week before the MPO assay was performed. MPO activity was determined using an assay described previously, ${ }^{21}$ but with the volumes of each reagent modified for use in 96 well microtitre plates. The rate of change in absorbance at $450 \mathrm{~nm}$ over a $90 \mathrm{sec}-$ ond period was determined using a kinetic microplate reader (Molecular Devices, Canada). One unit of MPO activity was defined as that degrading one micromole of hydrogen peroxide per minute at $25^{\circ} \mathrm{C}$. Values are expressed as units of MPO activity per milligram of tissue sampled (U/mg tissue).

COLONIC EPITHELIAL PERMEABILITY

To provide further a quantitative measure of epithelial dysfunction, in separate experiments, colonic epithelial permeability was determined in inflamed wild type or iNOS deficient mice at 24 hours, 72 hours, and seven days after TNBS treatment. Healthy mice from both groups were studied as controls. Mice were anaesthetised by intraperitoneal injection with a mixture of ketamine hydrochloride $200 \mathrm{mg} / \mathrm{kg}$ (Rogar/STB Inc., Montreal, Quebec, Canada) and xylazine $10 \mathrm{mg} / \mathrm{kg}$ (MTC Pharmaceuticals, Cambridge, Ontario, Canada). The left jugular vein was cannulated to administer anaesthetic and a laparotomy was performed. Both renal pedicles were ligated. The entire large intestine was isolated by fitting an inflow tube at the ascending colon-caecal junction and an outflow tube from the anus. The colonic loop was perfused with warm Tyrodes solution.

Chromium-51 labelled EDTA was injected intravenously to obtain counts per minute of at least $25000 / \mathrm{ml}(100-150 \mu \mathrm{Ci} / \mathrm{kg}) .{ }^{51} \mathrm{Cr}-\mathrm{EDTA}$ is a 300 molecular weight marker which is restricted by the mucosal barrier but not the vasculature. ${ }^{22}$ Therefore, any movement of ${ }^{51} \mathrm{Cr}$-EDTA is a direct reflection of changes across the mucosal not vascular barrier. ${ }^{51} \mathrm{Cr}$-EDTA activity in plasma and $2 \mathrm{ml}$ aliquots of perfusate was measured in an autogamma counter (WIZARD 3", model 1480; Wallac Oy, Turku, Finland). At the end of the experiment the colonic loop was removed, rinsed and weighed. The plasma to lumen clearance of ${ }^{51} \mathrm{Cr}$-EDTA was calculated as follows:

$$
\text { Clearance }=\frac{\mathrm{cpm}_{\mathrm{p}} \times \mathrm{pr} \times 100}{\mathrm{cpm}_{\mathrm{pl}} \times \mathrm{wt}}
$$

where clearance of ${ }^{51} \mathrm{Cr}$-EDTA is given in $\mathrm{ml} / \mathrm{min} / 100 \mathrm{~g}, \mathrm{cpm}_{\mathrm{p}}$ is counts $/ \mathrm{min} / \mathrm{ml}$ perfusate, $\mathrm{pr}$ is the perfusion rate, $\mathrm{cpm}_{\mathrm{pl}}$ is counts/ $\mathrm{min} / \mathrm{ml}$ plasma, and wt is weight of the intestinal segment in g. The animals were allowed a 30 minute control period to equilibrate after ${ }^{51}$ Cr-EDTA administration. Samples of perfusate were collected every 10 minutes for one hour and the average ${ }^{51} \mathrm{Cr}$-EDTA clearance was obtained.

IMMUNOHISTOCHEMISTRY

Colonic samples of tissue seven days postTNBS were used for immunohistochemistry; they were fixed overnight in Zamboni's fixative $(\mathrm{pH} 7.4)$ at $4^{\circ} \mathrm{C}$, rinsed $(3 \times 10$ minutes $)$ in phosphate buffered saline (PBS), transferred to

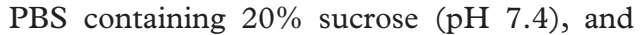
stored at $4^{\circ} \mathrm{C}$ overnight. They were embedded in OCT embedding medium (Sakura Finetek Inc., Torrance, California, USA), cryostat sectioned at $12 \mu \mathrm{m}$, thaw mounted onto poly-Dlysine coated slides, and dried.

To assess the expression of iNOS protein in colonic tissue, frozen sections were rehydrated in PBS containing $0.1 \%$ Triton X-100 (PBS$\mathrm{T}$ ), and incubated with $2 \%$ normal goat serum in PBS for 30 minutes at room temperature, to block non-specific binding, before being placed in primary antibody. Sections were then incubated for 48 hours at $4^{\circ} \mathrm{C}$ with a rabbit polyclonal antibody raised against iNOS (1/ 500; N32030, Transduction Laboratories, Lexington, Kentucky). The specificity of iNOS antibody was verified by substitution of the same concentration of normal rabbit immu- 
A

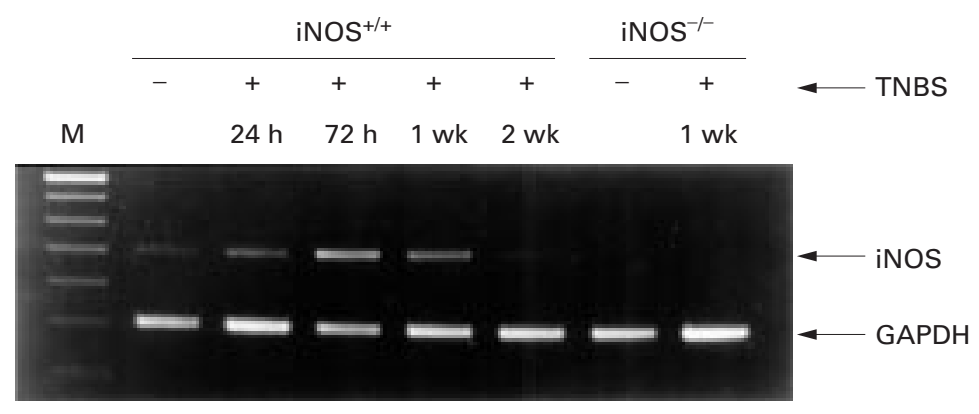

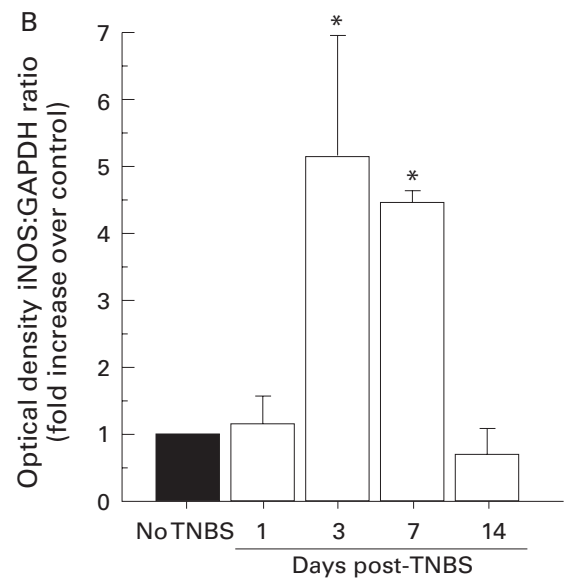

Figure 1 (A) A representative 2\% agarose gel of RT-PCR products. Base pair markers (M) denoting DNA size are shown in the far left lane. Glyceraldehyde-3-phosphate dehydrogenase (GAPDH; internal marker) and inducible nitric oxide synthase (iNOS) RT-PCR products are shown for non-inflamed (without trinitrobenzene sulphonic acid $(-T N B S)$ ) or inflamed $(+T N B S)$ wild type $\left(i N O S^{++}\right)$and $i N O S$ deficient $\left(i N O S^{-1}\right)$ tissue. $(B)$ Mean data $(n=4)$ after semiquantitative analysis of the iNOS band intensity expressed as a ratio of the GAPDH band for the various groups. The intensity of $i N O S$ band in non-inflamed mice was equated to one and the other bands expressed as fold increase over this level. *Significantly greater than wild type non-inflamed group.

noglobulin $\mathrm{G}(\mathrm{IgG})$ as was used for the anti-iNOS IgG.

To assess the possible cellular source of iNOS, sections were incubated with primary antibody for iNOS (as above) in combination with a monoclonal antibody raised in rat against mouse neutrophils (1/500; clone $7 / 4$, AMU0021, Camarillo, California) or a monoclonal antibody raised in rat which recognises tissue macrophages (1/50 clone M1/70; MCA 74, Serotec Ltd, Kidlington, Oxford, UK, which recognises Mac-1 differentiation antigen "CD11b"). The monoclonal rat antimouse CD11b antibody primarily recognises macrophages. ${ }^{23}$ The monoclonal rat antimouse neutrophil (polymorphic) antibody labels specifically all neutrophils and does not stain inflammatory macrophages, lymphocytes, eosinophils, mast cells, and mature erythroid cells in haemopoietic or lymphoid tissues. ${ }^{24}$ All antibodies were diluted in PBS-T containing $0.1 \%$ bovine serum albumin. Immunohistochemical controls routinely performed involved incubation with blocking solution and diluent in place of the primary antibody.

Sections were rinsed $(3 \times 10$ minutes $)$ with PBS-T and incubated for one hour at room temperature with sheep antirabbit IgG conjugated to CY3 (1/100) alone or combined with donkey antirat IgG conjugated to fluorescein isothiocyanate (FITC, 1/50). After a final wash $(3 \times 10$ minutes) with PBS-T, sections were mounted in bicarbonate buffered glycerol $(\mathrm{pH}$ 8.6) and examined using a Zeiss Axioplan fluorescence microscope. Sections were photographed using Kodak TMax film.

\section{STATISTICAL ANALYSIS}

Data are expressed as mean (SEM). Groups of data were compared using the non-parametric Mann-Whitney U test or Kruskal-Wallis one way analysis of variance (ANOVA) followed by a Dunns multiple comparison test. Probabilities of $5 \%$ or less were considered statistically significant.

\section{Results}

INOS MRNA EXPRESSION

Levels of iNOS mRNA were measured by RT-PCR in wild type non-inflamed and inflamed mice at 24 and 72 hours, and seven and 14 days after TNBS treatment. Noninflamed and inflamed iNOS deficient mice were also studied at seven days. Figure 1A shows a representative band for each time point in wild type mice. Interestingly, some message for iNOS was observed in untreated wild type mice. The RT-PCR product bands were quantified using non-linear densitometry, and the ratio of the iNOS to internal standard (GAPDH) was determined. The mean optical density ratio of wild type mice that did not receive TNBS $(n=4)$ was taken as 1 and each band expressed as a fold increase of this mean. Figure 1B shows the mean fold increase for four mice. At 24 hours after TNBS administration no significant increase in iNOS message was observed relative to control. However, by 72 hours post-TNBS administration there was a fivefold increase in the mean iNOS mRNA levels in wild type mice. This level was maintained at seven days $(\mathrm{p}<0.05)$, highlighting the dramatic increase in iNOS at this time point. Messenger RNA levels had returned to control values by 14 days. No detectable levels of RT-PCR products were observed in TNBS treated (seven days) and untreated iNOS deficient mice (fig 1A).

\section{ACUTE COLITIS: FIRST 72 HOURS}

Figure 2 illustrates macroscopic damage scores and MPO activity in wild type and iNOS deficient mice, 24 and 72 hours after TNBS administration. A significant increase in macroscopic damage score and granulocyte infiltration was observed at both time points in wild type mice. In iNOS deficient animals, macroscopic damage score (fig 2A) was significantly increased compared with their wild type counterparts at 24 hours, although granulocyte infiltration (fig 2B) was not significantly different. These data correlate well with data previously reported by our laboratory in an 

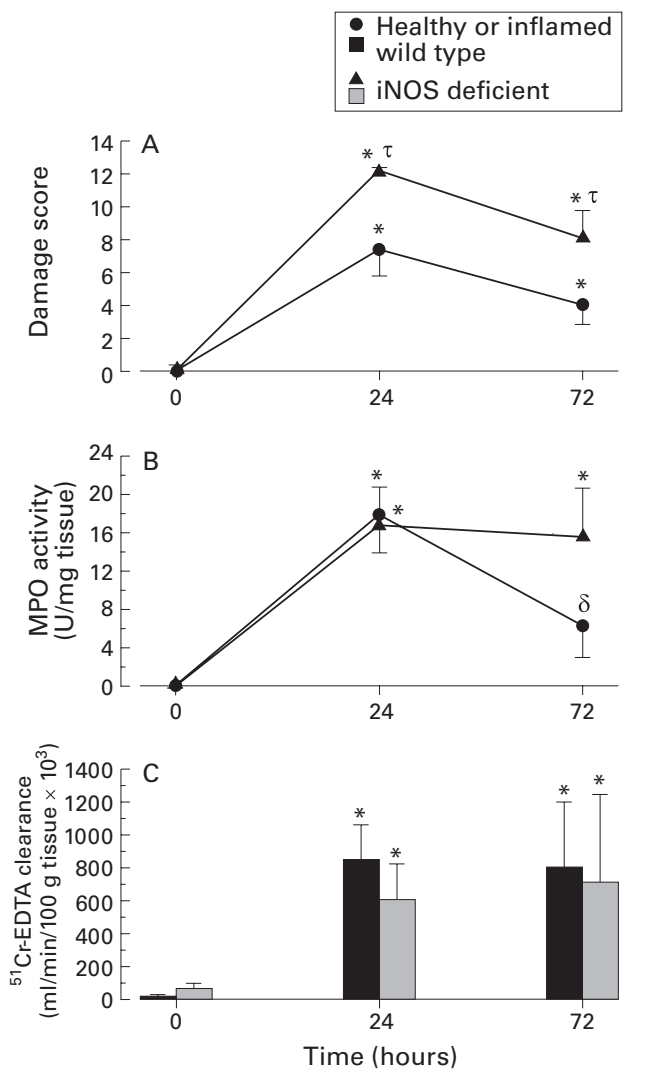

Figure 2 (A) Macroscopic damage score, (B) myeloperoxidase (MPO) activity, and (C) blood to lumen leakage of ${ }^{51} \mathrm{Cr}$-EDTA leakage as an indication of mucosal permeability in colonic tissue. $n \geqslant 6$ in all groups.

* Significant increase from healthy mice; $\delta$ significant

decrease from 24 hour time point; $\tau$ significant increase

from wild type mice. iNOS, inducible nitric oxide synthase.

acute intestinal inflammatory response (acetic acid induced colitis in mice) ${ }^{25}$ At this time point colonic damage has been shown to be independent of granulocyte infiltration in acetic acid induced ${ }^{26}$ and TNBS induced colitis. ${ }^{27}$ Substantial granulocyte infiltration was confirmed histologically with both wild type and iNOS deficient mice. Significant destruction of mucosal architecture (including epithelial cell layer), goblet cell depletion, and mucosal and submucosal oedema were also noted. Histological scoring of this tissue in wild type and iNOS deficient groups yielded mean scores of $7.5(0.2)$ and $8(0)$ respectively (see table 2 ).

Macroscopic damage remains significantly elevated in iNOS deficient mice 72 hours after the induction of colitis compared with the wild type inflamed group. At this time, granulocyte infiltration remained elevated in iNOS deficient mice; however, in wild type mice there

Table 2 Histological scores for wild type and inducible nitric oxide synthase (iNOS) deficient mice, 24 and 72 hours, and seven days after administration of trinitrobenzene sulphonic acid (TNBS)

\begin{tabular}{lll}
\hline Time point & Wild type & iNOS deficient \\
\hline Untreated & $0.3(0.2)$ & $0.2(0.2)$ \\
TNBS-24 h & $7.5(0.2)^{\star}$ & $8.0(0)^{\star}$ \\
TNBS-72 h & $4.8(1.0)^{\star}$ & $5.1(0.7)^{\star}$ \\
TNBS-7 days & $6.4(1.0)^{\star}$ & $7.9(1.0)^{\star}$ \\
\hline
\end{tabular}

$n \geqslant 5$. ${ }^{*}$ Significant increase from untreated groups. No significant differences in histological scores were observed between the two groups.
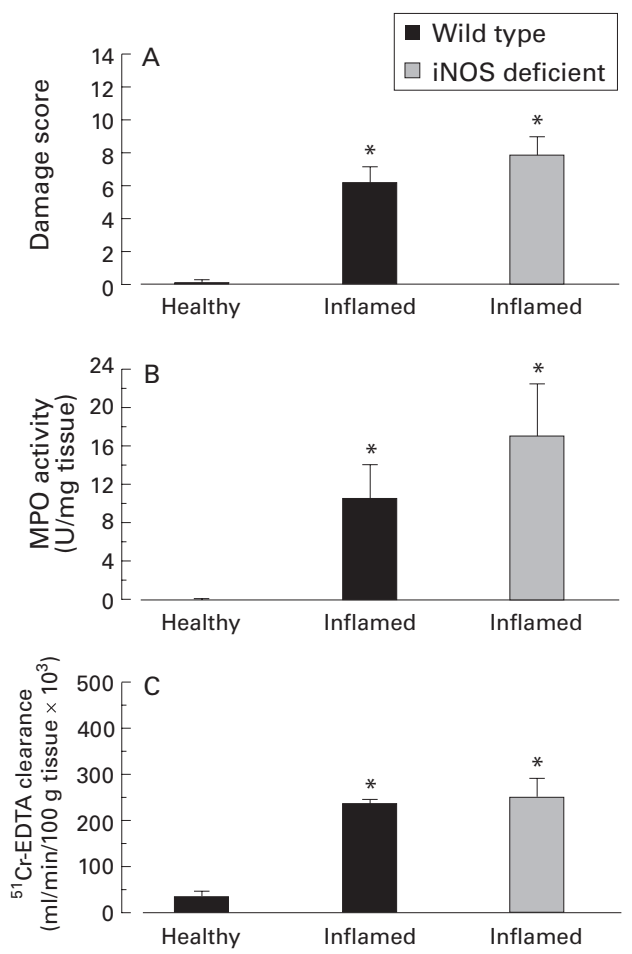

Figure 3 (A) Macroscopic damage score, (B) myeloperoxidase activity (MPO), and (C) blood to lumen leakage of ${ }^{51} \mathrm{Cr}$-EDTA leakage as an indication of mucosal permeability in colonic tissue from healthy or inflamed wild type or inducible nitric oxide synthase (iNOS) deficient mice. $n \geqslant 6$ in all groups. *Significant increase from healthy mice. There were no significant differences between iNOS deficient and wild type animals after seven days of inflammation.

was a significant drop in the level of neutrophil infiltration into colonic tissue (although this was not significantly different from iNOS deficient mice at this time point). Transmural cellular infiltration was observed histologically with crypt abscess formation and mild to moderate loss of mucosal architecture. Haemorrhage was also noted in the mucosa in areas of frank ulceration. Mean histological scores from tissue sections from wild type and iNOS deficient mice were 4.8 (1.0) and 5.1 (0.7) respectively (table 2 ). These histological scores show that sections of tissue from both wild type and iNOS deficient have a similar degree of inflammation. It is extremely important to note that histological score does not reflect the extent of damage along the length of colon which was much larger as assessed macroscopically (fig 2A). For example, the mean length of tissue involved in iNOS deficient mice was $2-2.5 \mathrm{~cm}$ whereas only $0.5 \mathrm{~cm}$ was observed in wild type tissue.

Next, we used the technique of blood to lumen clearance of ${ }^{51} \mathrm{Cr}$-EDTA to measure mucosal permeability. A very large elevation in ${ }^{51} \mathrm{Cr}$-EDTA leakage was observed in both wild type and iNOS deficient mice 24 hours post-TNBS administration (fig 2C). This level of mucosal dysfunction was maintained at 72 hours. No significant differences in mucosal permeability were observed in non-inflamed or inflamed wild type and iNOS deficient mice at either time point. This is not surprising as frank 

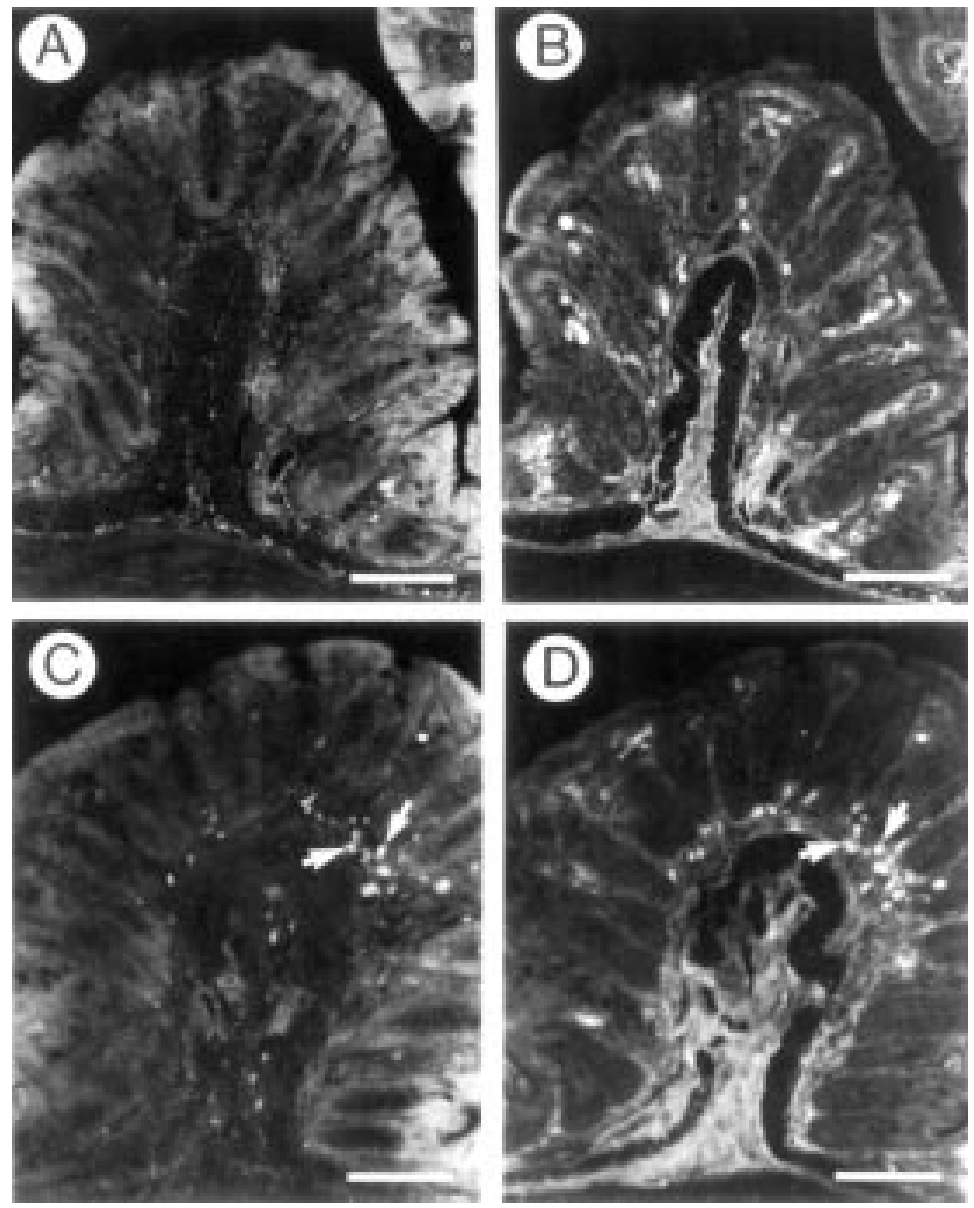

Figure 4 Fluorescence micrographs of inducible nitric oxide (iNOS) immunoreactivity ( $A$ and $C$ ) in healthy wild type animals. The same sections were double labelled to examine the cellular source of $i N O S$ (B and D). Little or no iNOS was present in normal animals in macrophages (B). However, occasional small clusters of iNOS immunoreactive neutrophils (D) were observed in otherwise healthy tissue. Scale bar: $100 \mu \mathrm{m}$.

haemorrhage suggests no restriction to blood components across the mucosa at these time points.

\section{CHRONIC COLITIS}

TNBS induced inflammation develops into a chronic phase by seven days onwards in rats ${ }^{20} 28$ and mice. ${ }^{16}{ }^{17}$ In this model mast cell hyperplasia, macrophages, and lymphocytes were noted up to two weeks post-TNBS. ${ }^{16}$ In this study we observed a 5.5-fold increase in macrophage numbers over non-inflamed control tissue seven days post-TNBS administration (see figs $4 \mathrm{~B}$ and 6B). Figure 3 illustrates macroscopic damage scores (fig 3A) and myeloperoxidase activity (fig 3B) in wild type and iNOS deficient mice seven days after TNBS treatment. In wild type mice a significant, 12-fold increase in macroscopic damage score was observed over that in healthy controls. This damage score represents the presence of at least two or more areas of ulceration, diarrhoea, and occasional adhesions and was accompanied by a significant elevation in granulocyte infiltration (fig 3B).

Histologically, a significant amount of damage was observed in both iNOS deficient and wild type groups (table 2). Tissue sections displayed varying degrees of damage ranging from complete destruction of the mucosal surface with muscle thickening, transmural cellular infiltration, and the presence of granulomas, to areas of distinct ulceration, with cellular infiltration and goblet cell depletion limited to the surrounding tissue. The extent of damage as measured by macroscopic damage scores was similar in both groups. No significant differences were observed in damage (macroscopic or microscopic) or granulocyte infiltration at this time point in iNOS deficient mice compared with inflamed wild type mice despite high levels of iNOS mRNA. As no frank haemorrhage was noted macroscopically some barrier function was regained. Indeed, the mean value for ${ }^{51} \mathrm{Cr}$-EDTA leakage into the lumen of the bowel was $65 \%$ lower $(\mathrm{p}<0.05)$ at seven days (fig 3C) after TNBS treatment than at 72 hours (fig 2C). Nevertheless, a sevenfold increase in mucosal permeability in inflamed wild type mice was noted compared with healthy controls (fig 3C). This highly sensitive quantitative assay of mucosal permeability revealed no difference between iNOS deficient and wild type mice; a significant, and equally large, increase in mucosal permeability was observed in iNOS deficient mice.

Therefore seven days post-TNBS, iNOS deficient mice developed a similar level of colonic damage and dysfunction (as measured by macroscopic damage, microscopic damage, cellular infiltration, and epithelial permeability) despite the inability to produce NO from iNOS.

TNBS induced inflammation was also studied in wild type and iNOS deficient mice 14 days after TNBS treatment. As previously indicated there was no increase in message for iNOS at this time point post-TNBS. Only very mild ulceration, no stricture formation or diarrhoea (3.2 (1.3)) and little neutrophil infiltration (7.6 (4.4) U/mg tissue) was observed at 14 days in wild type mice. No significant differences were observed in these parameters between wild type and iNOS deficient mice at this time point (damage score 1.7 (1.7), $\mathrm{p}=0.28$; MPO activity, $2.2(0.9) \mathrm{U} / \mathrm{mg}$ tissue, $\mathrm{p}=0.25$ ), suggesting that the early enhanced injury in iNOS deficient animals, did not impact on the development of TNBS induced inflammation between seven and 14 days.

\section{IMMUNOHISTOCHEMISTRY}

Figure 4A and C illustrates inducible NOS immunoreactivity in healthy, wild type mouse colon. It is clear that in healthy (macroscopic damage scores, MPO activity, and histology were all normal) mouse colon there were one or two very small areas of iNOS protein expression. Weakly immunoreactive epithelial cells and occasional patches of immunoreactive cells in the mucosal lamina propria were consistently observed in normal tissue. These data correlate well with a small amount of message for iNOS in healthy wild type mice. Double labelling for tissue macrophages (fig 4B) or neutrophils (fig 4D) revealed that the few iNOS positive cells in the lamina propria were neutrophils. Resident populations of macrophages in the healthy colon did not express iNOS immunoreactivity under normal conditions. The small amount of 

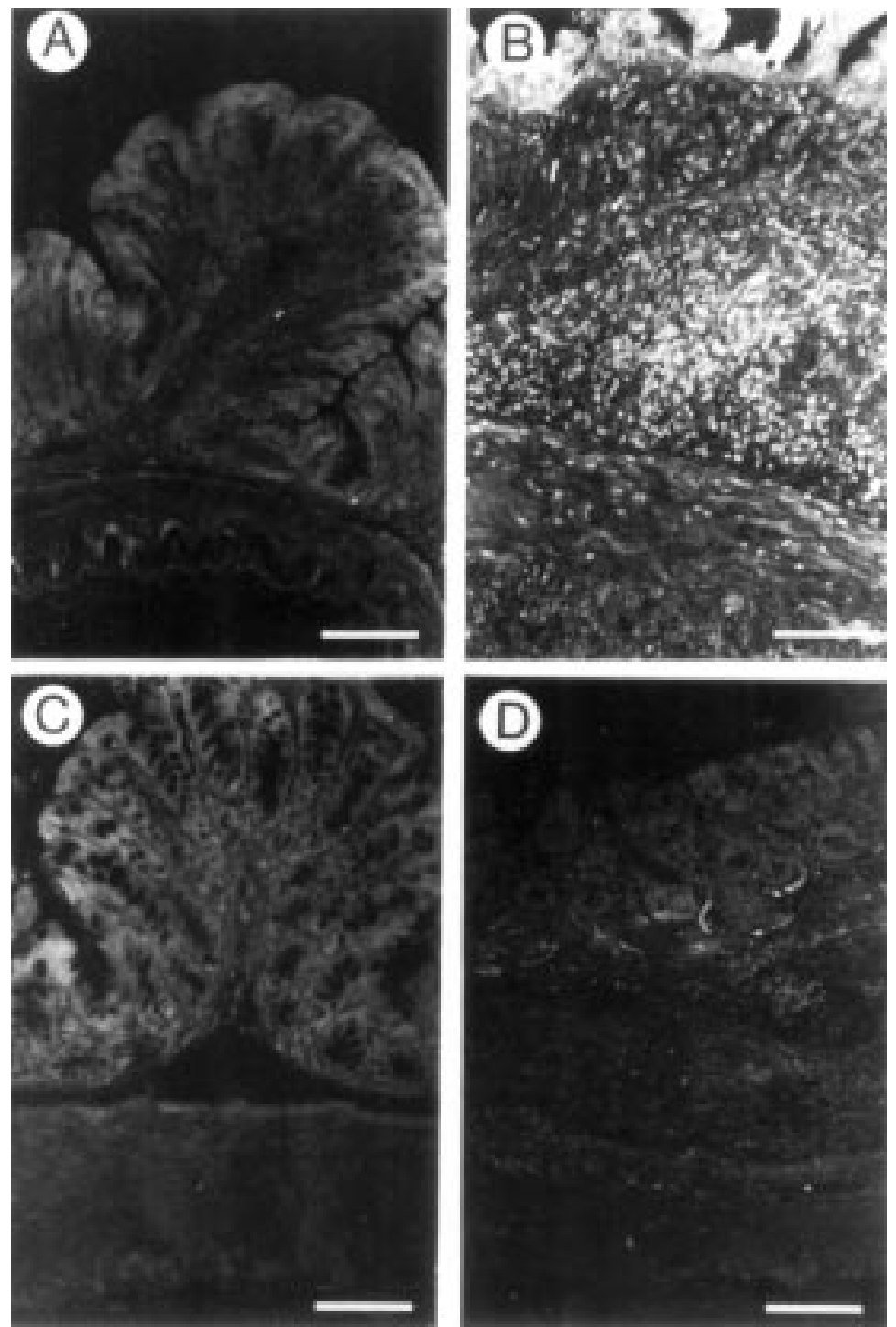

Figure 5 Fluorescence micrographs of inducible nitric oxide (iNOS) immunoreactivity in representative sections from a healthy wild type mouse $(A)$, healthy iNOS deficient mouse (C), inflamed wild type mouse (B), and inflamed iNOS deficient mouse (D). There was a substantial increase in iNOS immunoreactivity in inflamed mouse colon seven days after treatment with trinitrobenzene sulphonic acid in wild type mice (B). No iNOS

immunoreactivity was observed in the $i N O S$ deficient mice (C and D). Scale bar: $100 \mu \mathrm{m}$.

iNOS in the colon is not surprising as the colon is always exposed to an abundance of bacteria.

Figure 5A and B illustrates iNOS immunoreactivity in normal (fig $5 \mathrm{~A}$ ) and inflamed (fig 5B) colon seven days after TNBS treatment. These data illustrate an impressive increase in iNOS immunoreactivity in mouse colon seven days after TNBS treatment. Moreover, iNOS deficient mice provided a useful control to support the specificity of the staining in the wild type animals. There was no iNOS immunoreactivity in iNOS deficient mice under basal or inflamed conditions (fig $5 \mathrm{C}$ and $\mathrm{D}$ respectively). All other controls were negative, also supporting the specificity of the antibodies used in this study. The increase in iNOS immunoreactivity in the wild type animal correlates well with the increased levels of iNOS mRNA which were measured using RT-PCR (fig 1).

The cellular source of iNOS was studied by double labelling immunohistochemistry. Fig- ure 6 illustrates the colocalisation of iNOS immunoreactivity with inflammatory cell infiltrate in wild type animals seven days after TNBS treatment. Though there was considerable overlap in the distribution of iNOS (fig 6A) and macrophages (fig 6B), macrophages were not the major source of iNOS immunoreactivity in inflamed tissues. In fact, neutrophils which were the most abundant infiltrating cells (fig 6D) were consistently found to express iNOS immunoreactivity (fig 6C). At higher magnification iNOS immunoreactivity (fig 6E) can clearly be seen within neutrophil labelled cell bodies (fig $6 \mathrm{~F}$ ).

\section{Discussion}

This study assessed the role of iNOS in the acute and chronic phases of TNBS induced colitis by using mice genetically deficient in the inducible isoform of NOS. The results from this study clearly show a statistical increase in iNOS messenger RNA in wild type mice 72 hours after TNBS treatment which was maintained for seven days. Although there was always some increase in iNOS message at 24 hours this was difficult to detect adequately using the GeneQuant spectrophotometer and underscores the dramatic increase in iNOS at the later time points. An increase in iNOS protein was also measured by immunohistochemistry seven days post-TNBS. By 14 days in this model of colitis there was no detectable message for iNOS. Concurrent with the elevation in iNOS expression in wild type mice, we were able to show an increase in ulceration, diarrhoea, and stricture formation and a destruction of the mucosal architecture in colonic tissue. This increased macroscopic and microscopic damage was accompanied by a striking increase in cellular infiltration and mucosal permeability as measured by blood to lumen leakage of ${ }^{51} \mathrm{Cr}$-EDTA.

In mice which are genetically manipulated to be deficient in the iNOS gene and therefore incapable of the high output production of NO during intestinal inflammation, there were clearly two phases to the inflammation. At the earlier time points, when the inflammation can still be regarded as acute, macroscopic damage scores were significantly increased in iNOS deficient mice relative to wild type mice. These data are similar to data published recently in the acetic acid induced model of intestinal inflammation which indicated that iNOS deficient mice have a reduced ability to resolve inflammation induced as a result of an acute insult. ${ }^{25}$ The NO production from iNOS may provide protection in the acute stages of inflammation in a similar manner as does the supplementation with nitric oxide donors (by reducing neutrophil adhesion). ${ }^{29-31}$ Recently, iNOS deficient mice showed increased levels of leucocyte adhesion in response to lipopolysaccharide (using intravital microscopy), suggesting that the inducible isoform of NOS is capable of functioning as a regulator of leucocyte recruitment, not unlike cNOS. ${ }^{32}$ In addition, nitric oxide can limit inflammation by free radical scavenging, ${ }^{33-35}$ and stabilising mast cells. $^{3637}$ It is possible that increased NO 

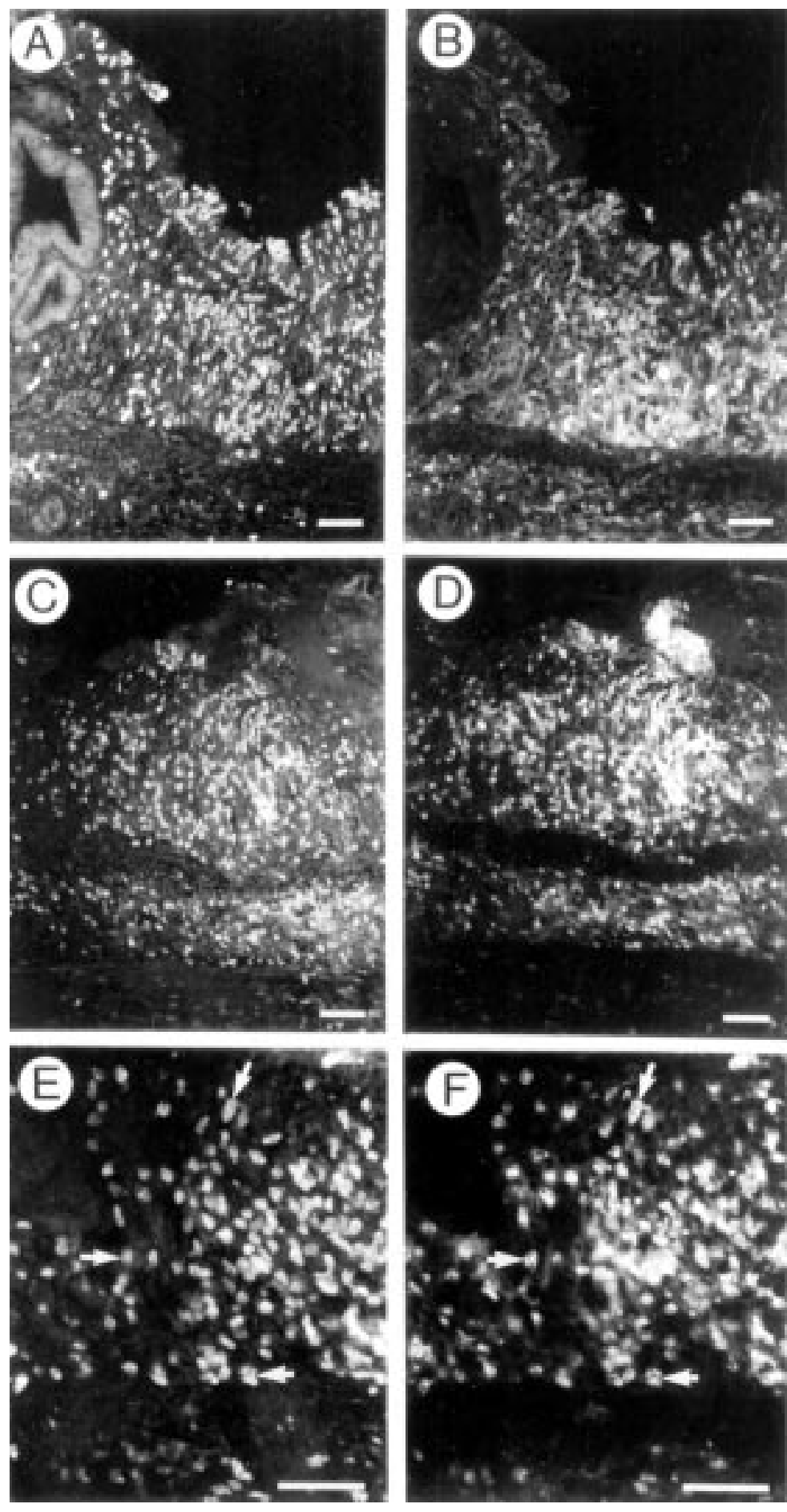

Figure 6 Fluorescence micrographs of inducible nitric oxide (iNOS) immunoreactivity $(A, C$, and $E)$ in wild type animals seven days after treatment with trinitrobenzene sulphonic acid. The same sections were double labelled to examine the cellular source of $i \operatorname{NOS}(B, D$, and $F)$. Though there was an overlapping distribution, there were relatively few macrophages that expressed iNOS immunoreactivity (B). In contrast, $i$ NOS immunoreactivity was extensively colocalised in neutrophils (D and F) in inflamed tissues. Scale bar: $100 \mu \mathrm{m}(A-D), 50 \mu \mathrm{m}$ (E and $F)$.

production from iNOS during this acute inflammatory stage acts to limit inflammation by one or more of these mechanisms.

Based on our previous work in the acetic acid induced model, it is tempting to predict that all models of intestinal inflammation will be exacerbated in the iNOS deficient mice. However, unlike the acetic acid induced model, the TNBS model does not resolve within seven days but develops from an acute to a chronic inflammation with the development of mast cell hyperplasia and infiltration of lymphocytes for at least two weeks. ${ }^{16}$ This model has significant infiltration of $\mathrm{CD}^{+} \mathrm{T}$ cells in the lamina propria, seven days post-TNBS administration, capable of increased production of interferon $\gamma$ and interleukins 2 and $4 .{ }^{17}$ In our study, during this phase of the inflammatory response (seven days post-treatment), the inflammation in iNOS deficient mice was identical to that observed in wild type mice.

A comprehensive approach was taken to try to uncover differences between the degree of intestinal damage induced in wild type or iNOS deficient mice. However, neither the extent of ulcerative area involved, presence of diarrhoea, stricture formation and adhesions, maximal bowel wall thickness, cellular infiltration, or microscopic tissue damage were different. In addition, the very sensitive index of intestinal permeability ( ${ }^{51} \mathrm{Cr}-\mathrm{EDTA}$ ), also revealed absolutely no difference between wild type and iNOS deficient mice. It should be noted that the level of ${ }^{51} \mathrm{Cr}$-EDTA leakage at this time point is significantly lower than that observed at earlier time points (approximately $65 \%$ lower) and therefore suggests the reestablishment of some barrier function in both groups of animals with no difference between groups.

These data differ considerably from inhibitor studies using NOS inhibitors given in the drinking water in experimental colitis where an attenuation of the inflammation was observed. ${ }^{5}{ }^{38}$ The discrepancy between these inhibitor studies and our studies in iNOS deficient mice may be twofold. Firstly, it is quite likely that some iNOS may be beneficial for bacterial killing ${ }^{39}$ and restitution, therefore complete inhibition of iNOS may be detrimental. With NOS inhibitors in drinking water, the degree of iNOS inhibition may be of lesser magnitude, so that some iNOS is still able to be used for essential functions (bacterial killing and restitution). Alternatively, continuous administration of an NOS inhibitor at the mucosal surface may inhibit NO production from both iNOS and the other NOS isoforms, which may be contributing to the deleterious effects of TNBS. Although less likely, this possibility cannot be excluded. In our study, complete and selective inhibition of iNOS proved not to be beneficial.

Interestingly, under normal, non-inflamed conditions, wild type mice were able to express a small amount of iNOS message and protein. This iNOS expression in healthy conditions was colocalised with neutrophils and epithelium in the colonic mucosa. It is of note that these animals express normal macroscopic and microscopic appearance, basal levels of neutrophils (MPO activity and immunohistochemistry), and normal intestinal permeability. It is conceivable that iNOS induced in neutrophils under normal conditions is a normal response to invading organisms and may be a method of maintaining homoeostasis in the intestine. It has been reported that iNOS 
is only induced in neutrophils after the transmigration out of the vasculature into the interstitium, ${ }^{40}$ consistent with our observations of the appearance of iNOS in neutrophils within the tissue, but not in the vasculature. To date, the induction of iNOS has been shown in numerous cell types in biopsy specimens from patients with ulcerative colitis or Crohn's disease including neutrophils, macrophages, epithelial cells, and endothelial cells. ${ }^{31-43}$ It should be noted, however, that iNOS immunoreactivity in biopsy specimens from patients with newly diagnosed inflammatory bowel disease $^{41}$ or from paediatric patients ${ }^{44}$ is detected exclusively in the epithelial cells while other studies detect iNOS in both epithelial cells and in the cellular infiltrate (neutrophils, mononuclear cells, and macrophages) into the lamina propria. ${ }^{3546}$ This difference in iNOS immunoreactivity may reflect disparate stages of intestinal inflammation from distinct patient populations. In this study, we showed that in the inflamed mouse colon the increased iNOS expression is due mostly to iNOS localised in neutrophils, with a lesser contribution from macrophages. In the iNOS deficient mice, there was no evidence of iNOS messenger RNA or iNOS protein. These animals served as an excellent control for our iNOS antibodies used in the immunohistochemical studies. Our data also suggest that in this model, epithelial and endothelial cells did not express iNOS, or did not express it to an extent that was detectable. This may reflect differences between this experimental model of intestinal inflammation in the mouse and clinical disease; however, it should be noted that there was limited epithelial restitution at this time point which may have limited our ability to detect iNOS in these cells in our study.

In conclusion, this study shows that iNOS is not required for the full development of chronic colitis in mice. Despite the absence of iNOS in iNOS deficient mice, an increase in mucosal permeability, macroscopic and microscopic damage, and granulocyte infiltration were induced to the same extent as in wild type mice. Even though damage was greater acutely in the iNOS deficient mice, this had little impact on the later development of disease in this model. Our data suggest that the iNOS is not essential for either the establishment or resolution of chronic intestinal inflammation associated with TNBS.

The authors acknowledge the technical assistance of Lesley Marshall and Christine Renz. We also thank Dr John S Mudgett of Merck Research Laboratories, Rathway, New Jersey for the supply of iNOS deficient mice. This study was supported by grants from the Crohn's and Colitis Foundation of Canada. D P Kubes is a Medical Research Council Scientist and Alberta Heritage Foundation for Medical Research (AHFMR) Senior Scholar. Dr KA Sharkey is an AHFMR Senior Scholar and Dr M Miampamba was an AHFMR Fellow.

1 Roediger We, Lawson MJ, Nance $\mathrm{SH}$, et al. Detectable colonic nitrite levels in inflammatory bowel diseasemucosal or bacterial malfunction? Digestion 1986;35:199204.

2 Boughton-Smith NK, Evans SM, Hawkey CJ, et al. Nitric oxide synthase activity in ulcerative colitis and Crohn's disease. Lancet 1993;342:338-40.

3 Singer II, Kawka DW, Scott S, et al. Expression of inducible Singer II, Kawka DW, Scott S, et al. Expression of inducible
nitric oxide synthase and nitrotyrosine in colonic epithenitric oxide synthase and nitrotyrosine in colonic epithe-
lium in inflammatory bowel disease. Gastroenterology 1996; 111:871-85.
4 Nathan C, Xie O-W. Nitric oxide synthases: roles, tolls and controls. Cell 1994;78:915-18.

5 Rachmilewitz D, Karmeli F, Okon E, et al. Experimental colitis is ameliorated by inhibition of nitric oxide synthase activity. Gut 1995;37:247-55.

6 Grisham MB, Specian RD, Zimmerman TE. Effects of nitric oxide synthase inhibition on the pathophysiology observed in a model of chronic granulomatous colitis. 7 Pharm Exp Ther 1994;271:1114-21.

7 Miller MJS, Sadowska-Krowicka H, Chotinaruemol S, et al. Amelioration of chronic ileitis by nitric oxide synthase inhibition. F Pharm Exp Ther 1993;264:11-16.

8 Ribbons KA, Clark DA, Currie MG, et al. Inducible nitric oxide synthase and idiopathic colitis in rhesus macaques [abstract]. Gastroenterology 1995;108:A903.

9 Hogaboam CM, Jacobson K, Collins SM, et al. The selective beneficial effects of nitric oxide inhibition in experimental colitis. Am f Physiol 1995;268:G673-84.

10 Conner EM, Chen Y, Grisham MB. Effect of nitric oxide synthase (NOS) inhibition on dextran sulfate sodium (DSS)-induced colitis in rats and mice [abstract]. Gastroenterology 1995;108:A801.

11 Pfeiffer CJ, Qui BS. Effects of chronic nitric oxide synthase inhibition on TNB-induced colitis in rats. 7 Pharm Pharmacol 1995;47:827-32.

12 Misko TP, Moore WM, Kasten TP, et al. Selective inhibition of the inducible nitric oxide synthase by aminoguanidine. Eur F Pharmacol 1993;233:119-25.

13 Lopez-Belmonte J, Whittle BJR. Aminoguanidine-provoked leukocyte adherence to rat mesenteric venules: role of constitutive nitric oxide synthase inhibition. $\mathrm{Br}$ F Pharmacol 1995;116:2710-14.

14 Ohrui $\mathrm{T}$, Yamauchi $\mathrm{K}$, Sekizawa $\mathrm{K}$, et al. Histamine $\mathrm{N}$-methyl transferase controls the contractile response of guinea pig trachea to histamine. F Pharm Exp Ther guinea pig trachea

15 MacMicking JD, Nathan C, Hom G, et al. Altered responses to bacterial infection and endotoxic shock in mice lacking inducible nitric oxide synthase. Cell 1995;81:641-50.

16 Chin KW, Barrett KE. Mast cells are not essential to inflammation in murine model of colitis. Dig Dis Sci 1994;39: 513-25.

17 Neurath MF, Fuss I, Kelsall BL, et al. Antibodies to interleukin-12 abrogate established experimental colitis in mice. 7 Exp Med 1995;182:1281-90.

18 Chomczynski P, Sacchi N. Single-step method of RNA isolation by acid guanidium tiocyanate-phenol-chloroform lation by acid guanidium tiocyanate-phen

19 Wong H, Anderson WD, Cheng T, et al. Monitoring mRNA expression by polymerase chain reaction. The "primer dropping method". Anal Biochem 1994;223:251-8.

20 Morris GP, Beck PL, Herridge MS, et al. Hapten-induced model of chronic inflammation and ulceration in the rat colon. Gastroenterology 1989;96:795-803.

21 Krawisz JE, Sharon P, Stenson WF. Quantative assay for inflammation using myeloperoxidase assay. Gastroenterology 1984;87:1344-50.

22 Kubes P. Nitric oxide modulates epithelial permeability in the feline small intestine. Am $\mathcal{F}$ Physiol 1992;262:G1138-42.

23 Springer T, Galfre G, Secher DS, et al. Mac-1: macrophage differentiation antigen identified by monoclonal antibody. Eur 7 Immunol 1979;9:301-6.

24 Hirsch S, Gordon S. Polymorphic expression of a neutrophil differentiation antigen revealed by monoclonal antibody 7/4. Immunogenetics 1983;18:229-39.

25 McCafferty D, Mudgett JS, Swain MG, et al. Inducible nitric oxide synthase plays a critical role in resolving intestinal inflammation. Gastroenterology 1997;112:11022-7.

26 Yamada T, Zimmerman BJ, Specian RD, et al. Role of neutrophils in acetic acid-induced colitis in rats. Inflammation 1991;15:399-411.

27 Buell MG, Berin MC. Neutrophil-independence of the initiation of colonic injury. Comparison of results from three models of experimental colitis in the rat. Dig Dis Sci 1994; 39:2575-88

28 McCafferty D, Sharkey KA, Wallace JL. Beneficial effects of local or systemic lidocaine in experimental colitis. Am $\mathcal{F}$ Physiol 1994;266:G560-7.

29 Kubes P, Kurose I, Granger DN. NO donors prevent integrin-induced leukocyte adhesion, but not P-selectindependent rolling in postischemic venules. Am $\mathcal{F}$ Physiol 1994;267:H931-7.

30 Kubes P, Suzuki M, Granger DN. Nitric oxide: an endogenous modulator of leukocyte adhesion. Proc Natl Acad Sci USA 1991;88:4651-5.

31 Gaboury JP, Niu X-F, Kubes P. Nitric oxide inhibits numerous features of mast cell-induced inflammation. Circulation 1996;93:318-26.

32 Hickey MJ, Sharkey KA, Sihota E, et al. Inducible nitric oxide synthase-deficient mice have enhanced leukocyteendothelium interactions in endotoxemia. FASEB F 1997; 11:955-64.

33 Rubanyi GM, Ho EH, Cantor EH, et al. Cytoprotective function of nitric oxide: inactivation of superoxide radicals produced by human leukocytes. Biochem Biophys Res Commun 1991;181:1392-7.

34 Gaboury J, Woodman RC, Granger DN, et al. Nitric oxide prevents leukocyte adherence: role of superoxide. $A m \mathcal{F}$ Physiol 1993;265:H862-7.

35 Wink DA, Hanbauer I, Krishna MC, et al. Nitric oxide protects against cellular damage and cytotoxicity from reactive oxygen species. Proc Natl Acad Sci USA 1993;90:9813-17. 
36 Kanwar S, Wallace JL, Befus D, et al. Nitric oxide synthesis inhibition increases epithelial permeability via mast cells. inhibition increases epithelial pe

37 Kubes P, Kanwar S, Niu X-F, et al. Nitric oxide synthesis inhibition induces leukocyte adhesion via superoxide and mast cells. FASEB f 1993;7:1293-9.

38 Aiko S, Grisham MB. Spontaneous intestinal inflammation and nitric oxide metabolism in HLA-B27 transgenic rats. Gastroenterology 1995;109:142-50

39 Nathan CF, Hibb JB. Role of nitric oxide synthesis in macrophage antimicrobial activity. Curr Opin Immunol 1991;3 $65-70$.

40 Miles AM, Owens MW, Milligan S, et al. Nitric oxide synthase in circulating versus extravasated polymorphonuclear leukocytes. $\mathcal{F}$ Leukocyte Biol 1995;58:616-22.

41 Kolios G, Rooney N, Murphy CT, et al. Expression of inducible nitric oxide synthase activity in human colon epithelial cells: modulation by $\mathrm{T}$ lymphocyte derived cytokines. Gut 1998;43:56-63.
42 Ikeda I, Kasajima T, Ishiyama S, et al. Distribution of inducible nitric oxide synthase in ulcerative colitis. Am $\mathcal{F}$ Gastroenterol 1997;92:1339-41.

43 Iwashita E, Iwai A, Sawazaki J, et al. Activation of microvascular endothelial cells in active ulcerative colitis and detection of inducible nitric oxide synthase. $\mathcal{F}$ Clin Gastroenterol 1998;27:S74-9.

44 Levine JJ, Pettei MJ, Valderrama E, et al. Nitric oxide and inflammatory bowel disease: evidence for local intestinal production in children with active colonic disease. F Pediatr production in children with active color

45 Godkin AJ, De Belder AJ, Wong A, et al. Expression of nitric oxide synthase in ulcerative colitis. Eur $\mathcal{f}$ Clin Invest 1996;36:867-72.

46 Kimura H, Hokari R, Miura S, et al. Increased expression of an inducible isoform of nitric oxide synthase and the formation of peroxynitrite in colonic mucosa of patients with active ulcerative colitis. Gut 1998;42:180-7. 